\title{
Wireless Positioning System for Investigation of Road Traffic Accidents
}

\author{
Song Hong \\ Chongqing Key Lab of Traffic System \& Safety in \\ Mountain Cities \\ Chongqing, China \\ Traffic_engineer@sina.com \\ Ming Jianxiong
Chongqing Key Laboratory of Vehicle/Biology Crash
Chongqing, China
guanlantang@163.com \\ Tang Jun \\ Traffic and Patrol Police, Chongqing Police Department \\ Chongqing, China \\ 1073213894@qq.com
}

\author{
Zeng Ding \\ Traffic and Patrol Police, Chongqing Police Department \\ Chongqing, China \\ 125481765@qq.com \\ Duan Weijian \\ Traffic and Patrol Police, Chongqing Police Department \\ Chongqing, China \\ Duanweijian82@163.com \\ Yin Zhiyong \\ Chongqing Key Laboratory of Vehicle/Biology Crash \\ Chongqing, China \\ Zyyin@cta.cq.cn
}

\begin{abstract}
In traffic accident investigation, measurement and location of accident factors using traditional methods is a time-consuming work. Investigators must work on each measurement point to collect data, and then mark the position of the measured point in the scene graph. Due to the low efficiency of manual operation, the investigation time is long, thereby increasing the traffic recovery pressure. In order to solve the above-mentioned problem, this paper describes a wireless location system to graphically record the scene of road traffic accident. This system combines the ultrasonic technology and ZIGBEE wireless communication network, which firstly installs distance measuring equipments at all the measurement points including two reference points and other ones, and then completes the measurement work in one time. Use of ultrasonic technique guarantees the accuracy of distance measurement while ZIGBEE wireless communication network facilitates remote control device. The new system saves a lot of time spent on moving back and forth of various equipments and manual operation, thus improving the investigation efficiency.
\end{abstract}

Keywords- Road Traffic Accidents; Investigation; ZIGBEE; Ultrasonic Ranging; Wireless Positioning

\section{INTRODUCTION}

In the investigation of road traffic accidents, positioning of accident factors (such as vehicle, body, trace and so on) is a basic work, and also the basis of data for scene drawing. The conventional positioning methods include rectangular coordinate positioning, three point positioning and polar coordinate positioning ${ }^{[1][2]}$. Among them, polar coordinate method is rarely used in practical work. However no matter which method is used, the distance between the reference point and other measurement points should be collected. At present, tape is mainly used to measure each distance manually and then to record the data. At the same time, investigators have to draw the scene map. The cross of two processes not only affects the efficiency, but also brings many errors.

With the rapid growth of vehicle and highway mileage, road traffic pressure unceasingly is increasing and puts forward higher requirements on the traffic accident scene investigation and disposal. Therefore a new method and device to shorten the investigation time and improve efficiency has become an important direction of traffic accident research. Some researchers propose photography measurement to replace tape measurement ${ }^{[3][4]}$, but a calibration board is required to put in the scene, and the distant measurement points show low accuracy.

Wireless sensor network (WSN) in recent years has been widely used in indoor positioning system. It has the advantages of large coverage area, many measurement points and rapid positioning. But the detection accuracy of RSSI (Received Signal Strength Indicator) is 2-5 ${ }^{[5]}$, which is far from the required accuracy in traffic accident scene investigation (decimeter level precision). Considering the high precision of distance measurement of ultrasonic wave, we combine it with ZIGBEE wireless network to transmit control signal and data, so as to meet the practical needs in scene investigation of traffic accident. 


\section{APPARATUS AND METHOD}

\section{A. System structure}

As illustrated in Figure 1, the system consists of an ultrasonic transmission device, an ultrasonic receiving device, a wireless gateway, a notebook computer and a supporting pole. The transmitting and receiving devices are capable of communicating with the gateway over the ZIGBEE protocol ${ }^{[6]}{ }^{[7]}$. These devices and the wireless gateway are powered with a battery source and therefore can be used on the go. Transmitting device is mounted on the top of the support rod as the measurement reference point, to avoid any obstacle. At least two transmitting devices are required in order to archive positioning. The receiving devices are simultaneously used as the measurement points and can have many $(<50)$. The gateway has a temperature sensor ${ }^{[8]}$ that connects with the laptop via a serial port.

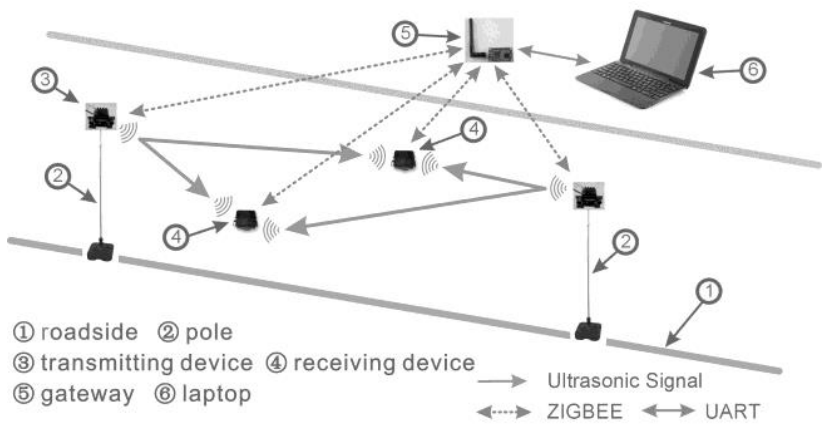

Figure 1. system block diagram

\section{B. Ranging principle}

As illustrated in Figure 2, laptop directs the wireless command to the gateway and the gateway then transmits a synchronization signal to a pair of transmitting and receiving device in time $t_{0}$. An ultrasonic signal is generated immediately after the transmission signal is received. The signals received in specified time is measured as $\mathrm{T} 1$ and the linear distance between two devices can be evaluated by a formula $\mathrm{s}=\mathrm{v} \times\left(\mathrm{t}_{1}-\mathrm{t}_{0}-\mathrm{t}_{\Delta}\right){ }^{[9]}$. Wherein, $\mathrm{t}_{\Delta} \neg$ is inherent time error in the system and can be obtained experimentally. The data is then sent from the receiver to the laptop via a gateway.

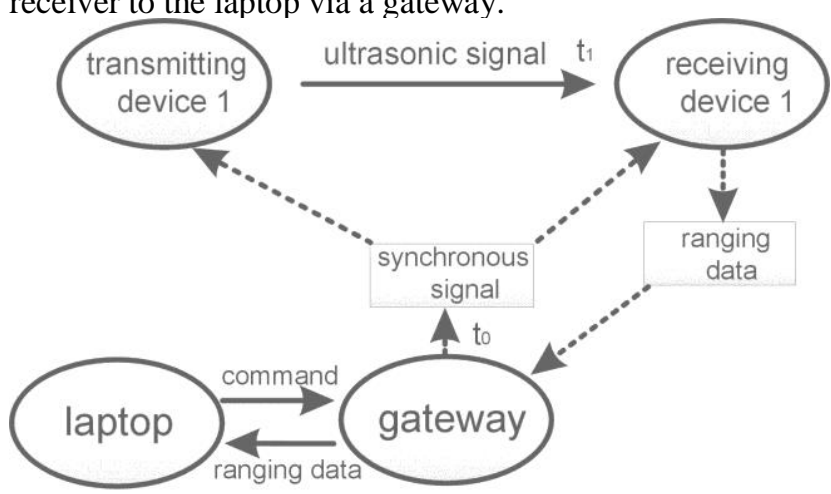

Figure 2. Single point ranging

\section{Multi-point measurement}

As illustrated in Figure 3, laptop uses gateway channel to send synchronizing signals to transmitting device 1 and receiving device $1^{[10]}$, and then receive measurement data 1 . It again sends synchronizing signals to transmitting device 2 and receiving device 1 , and then receives measurement data 2. Thus, we obtain the value of distance between the receiving apparatus 1 and the two transmitting devices. If more measuring points are required, the distance is always measured between the receiving device at each measurement point and the transmitting devices at the two reference points, conferring the above method.

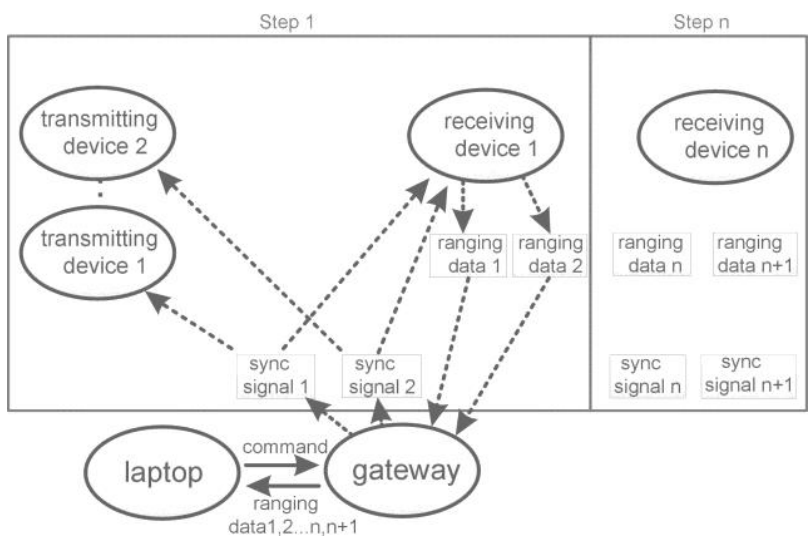

Figure 3. Multi-point ranging

\section{Positioning principle}

As illustrated in Figure 4, a significant height difference exists between transmitting and receiving device. Since, the height of transmitting device $\left(\mathrm{H}_{1}\right)$, receiving device $\left(\mathrm{H}_{2}\right)$ and the linear distance between the two devices $\left(\mathrm{L}_{1}\right)$ is known, the horizontal distance $\left(\mathrm{L}_{2}\right)$ can be obtained through the formula $l_{2}=\sqrt{l_{1}^{2}-\left(h_{1}-h_{2}\right)^{2}}$.

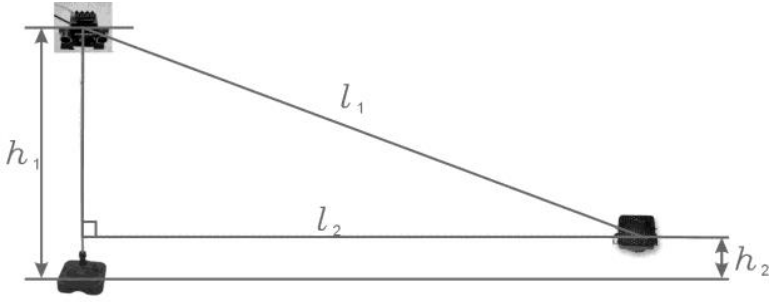

Figure 4. The actual distance

As illustrated in Figure 5, the distance between measurement point $M 1$ and reference points $B_{1}$ and $B_{2}\left(S_{1}\right.$ and $\mathrm{S}_{2}$ ) can be determined by ultrasonic detection; meanwhile the horizontal distance between two reference points $\left(\mathrm{S}_{3}\right)$ can be measured in advance

$\alpha_{1}=\operatorname{arcCos} \frac{S_{1}^{2}+S_{3}^{2}-S_{2}^{2}}{2 S_{1} S_{3}}, \quad \alpha_{2}=\operatorname{arcCos} \frac{S_{2}^{2}+S_{3}^{2}-S_{1}^{2}}{2 S_{2} S_{3}}$; thus angle $a_{1}$ and $a_{2}$ can be obtained by using the cosine theorem and the position relation of measurement point M1 and reference points B1 \& B2 can be determined. In 
the practical exploration, as shown in Figure $1, \mathrm{~B}_{1}, \mathrm{~B}_{2}$ are placed in the straight road edge and the distance between them is measured in advance. Special software on the laptop can quickly locate data from multiple measurement points by the above method.

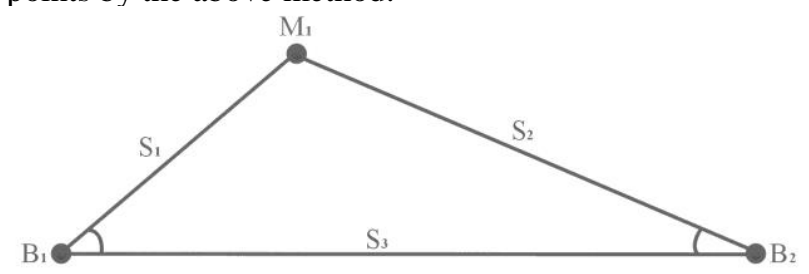

Figure 5. The cosine theorem

\section{E. Hardware design}

The CC2430 controllers made by TI corporation are set as the connecting nodes in the wireless communication system. The structure of terminal node circuit is illustrated in Figure 6. Processor, falsh rom, network transceiver and I/O interface are integrated in CC2430 chips, using slot to link the module for ultrasonic transmitting /receiving with circuit board of each node. Specific frequency pulsing signals are sent by CC2430 to transmitting module through I/O interface, which are converted into ultrasonic signals (D/A conversion) by transducer for transmitting afterward. Ultrasonic signals received by transducer from other devices are converted into pulsing signals by $\mathrm{A} / \mathrm{D}$ module and sent to I/O interface on CC2430. The processor of CC2430, instructed by program preinstalled in the flash rom, controls the module for ultrasonic transmitting / receiving and manipulates the network transceiver for communication. To enhance sensitivity of wireless communication in the field, external antenna is attached to terminal node, which is powered by 2 AA batteries. The voltage of $\mathrm{CC} 2430$ is $2.0-3.6 \mathrm{~V}$, which means it can be powered with the same source. The voltage for ultrasonic tansmitting/receiving is $5 \mathrm{~V}$, which needs voltage boost circuit. The disparity between terminal node and gateway is that there is no module for ultrasonic transmitting / receiving in gateway, whereas there is serial to USB port to connect gateway with laptop.

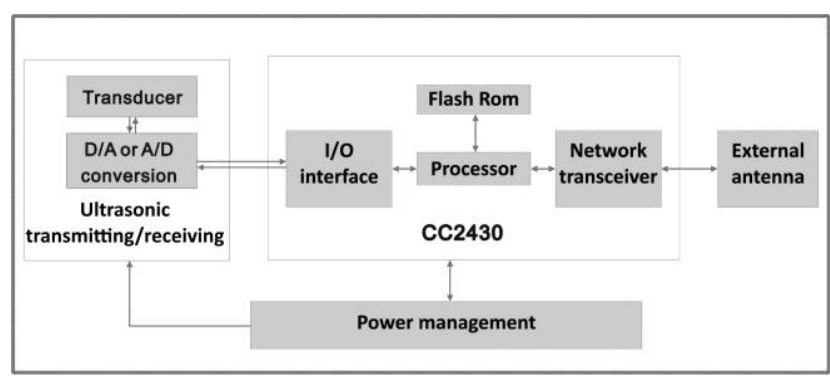

Figure 6. Terminal node circuit

Ultrasonic transmitting / receiving module shares the same set of circuit, but the tranducers for each are installed in different position, corresponding with their function. In order to enhance the measure distance, high voltage is necessary for transmitting module. For receiving module, the shape is specially designed to steadily receive the ultrasonic signals in range. Traditional threshold detction methods are inappropriate in long distance measurement, since strong interference to the ultrasonic signal from the outside. Specialized bit sequence is encoded as the pulsing signal for transmitting in the system, and related functions are installed in coding dignals and receiving signals to minimize the interference.

\section{F. Protocol stack}

Z-Stack protocol stack made by Texas Instruments Co. is adopted in software controlling the wireless terminals and gateway of the system. The whole wireless communicating system is a mode of terminal nodes directly connecting gateway (coordinator), which constitutes a star network. The coordinator should be powered on first, and will automatically set itself into selfintialization mode and then start to establish the network. After the network has been established, all powered-on terminal nodes is optioned to auto-find mode, search for the coordinator and join the network automatically. A solid bonding between terminal nodes and coordinator can be built up and further put into field processing.

The event processing of Z-Stack is illstrated in Figure 7. Operating System Abstraction Layer (OSAL) in Z-Stack is for multi-task processing ${ }^{[11]}$. The function of OSAL is to search for any event that need to be dealt with in CC2430. It will call coresponding event function to deal with if there is a event; or keep looking for. The OSAL in each terminal nodes detect any event in data receiving when gateway is sending instructions for measurement to a terminal node. The OSAL chooses to sending measurement signals or awaiting, accoding to different functions of modules. If two event happened simultaneously, (the terminal nodes are receiving the event of checking measurement data, for example), OSAL compare priorities of the two events and check the distance data after the processing of distance measurement being completed, because of the higher priority for measurement than that for checking. The program will reture to OSAL task cycle when all events have been finished.

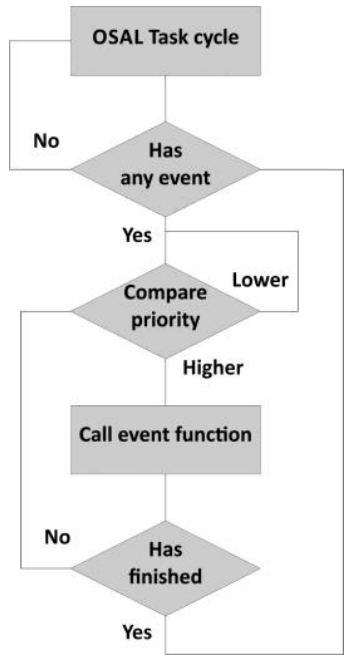

Figure 7. Event processing 


\section{ANALYSIS AND DISCUSSION}

The above-mentioned methods are based on the following assumptions: (1) the measurement region is a horizontal plane; (2) the scene size is within the allowable range of ultrasonic detection; (3) the ultrasonic velocity is a constant; (4) the ultrasonic transmitting and receiving angles satisfy the need of distribution in the field at any position. As for condition (1), the scene of traffic accident (such as highway) would be a flat slope in the worst case, rather than a wavy surface. Through a simple measurement of the road slope, the actual positioning data can be obtained using the trigonometric function. As for condition (2), the range of ultrasonic detection is about $20-40 \mathrm{~m}$ and more reference points can be installed in a larger scene, as illustrated in Figure 8. According to the presupposed distance, the reference points of $\mathrm{B} 1, \mathrm{~B} 2$ and $\mathrm{B} 3$ can be evenly placed to divide the scene into Zone1 and Zone2. Thereafter measurement points in two regions can be respectively positioned. In the end, the two regions are spliced.

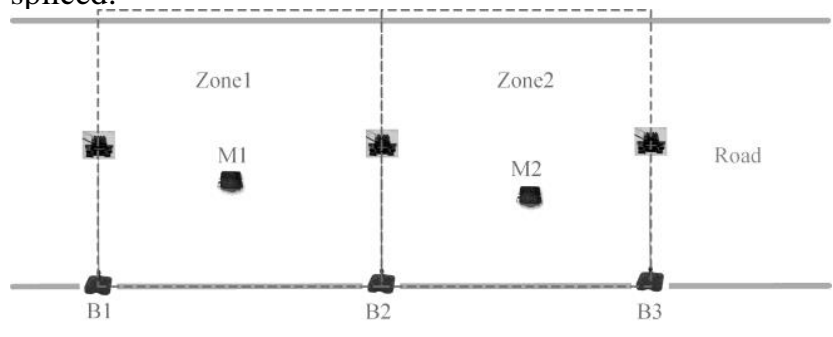

Figure 8. Scene mosaicing

For ultrasonic velocity, temperature is the main influencing factors under laboratory conditions, which can be corrected through a temperature sensor integrated in the gateway. In outdoor conditions, wind speed is an important factor to be considered; thereby a wind speed sensor is required. Moreover, further experiments should be conducted to establish its relationship with ultrasonic velocity. For condition (4), as illustrated in Figure 9, the ultrasonic transmission angle is normally $60^{\circ}$. If the distance between reference point $\mathrm{B} 1$ and $\mathrm{B} 2\left(\mathrm{~L}_{1}\right)$ is $40 \mathrm{~m}$, in three-lane road whose width $\left(\mathrm{W}_{1}\right)$ is usually $11.25 \mathrm{~m}$, the length of the area completely covered by the beam $\left(\mathrm{L}_{2}\right)$ can be calculated as $17 \mathrm{~m}$. If the road is narrowed to two lanes $\left(\mathrm{W}_{1}=7.5 \mathrm{~m}\right), \mathrm{L}_{2}$ will be extended to $31 \mathrm{~m}$. As a result, this system though has some technical problems that need further study, is fairly practical.

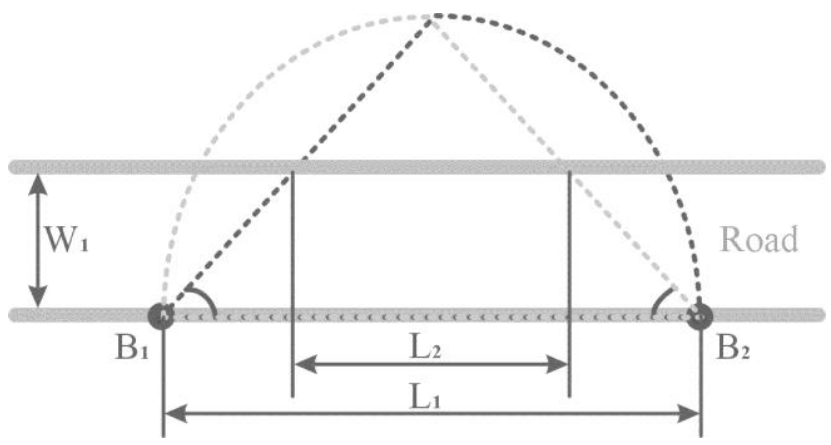

Figure 9. Beam range

\section{ACKNOWLEDGMENT}

This paper was supported by the open foundation of Chongqing Key Lab of Traffic System \& Safety in Mountain Cities(KTSS201201).

\section{REFERENCES}

[1] Xu Yi-gang. New Discussion of the Treatment of Road Traffic Accident $[\mathrm{M}]$. Shangdong: Shandong people's Publishing House, 2005.

[2] Ji Lei. Standardization Research for Scene Investigation of Road Traffic Accident Based on Computer.Xi'an: Chang'an University, 2010 .

[3] Tang Yang-san,Li jiang,Zhang jian,et al. Study on Camera Calibrating for Scene Measuring[J]. Journal of Changchun University of Science and Technology, 2005.9,28(3):64-66.

[4] Zhan Zong-qian, Zhang Jian-qing. Application of Digital Photogrammetry in Surveying and Mapping of the Traffic Accident Site[J]. 2006.1,23(1):147-150.

[5] Rabacy JJ, Ammer MJ , da Silva Jr. JL,Patel D, Roundy S.Picorodio support Ad Hoc Ultra-Low Power Wireles Networking[J]. Computer,2000,33(7):42-48.

[6] SUN Li-min, LI Jian-zhong, CHEN Yu, et a1. Wireless Sensor Networks[M]. Beijing:Tsinghua Univer-sity Press,2005.

[7] Li Xiao-wei,Xu Yong-jun,Ren Yuan-fen.Wireless Sensor Network Technology[M]. Beijing: Beijing Institute oF Press,2007.

[8] Zhao Hai-ming,Pu Yin-yong,Wang Ji-chan.Development of an Ultrasonic Distance Measurement System with High Precision[J] Mining R\&D,2006.6,26(3):62-65.

[9] Huang Jie,Wang Fu-bao, Duan Wei-jun. Ultrasonic Ranging Technology of WSN Nodes[J].Electronic Design Engineering, 2013.3,21(6):120-122

[10] Li Chang-lu,Su Han-song.An Ultrasonic Positioning System[J]. Reserch and Exploration in Laboratory,2013.2,32(2):39-44

[11] Li Li-li. Design and Implement of Wirless Sensor Network Node Based on ZIGBEE[D]. Sichuan: Southwest Jiaotong University, 2008 